\title{
A beneficial cardiometabolic health profile associated with dietary supplement use: A cross-sectional study
}

\author{
Paul F. Jacques@ and Gail Rogers \\ Jean Mayer USDA Human Nutrition Research Center on Aging, Tufts University, Boston, MA, USA
}

\begin{abstract}
As a follow-up to an earlier study demonstrating healthier cardiometabolic profiles among long-term multiple dietary supplement (LTMDS) users, we examined if cardiometabolic health benefits associated with LTMDS use persisted with aging. The study is based on LTMDS users from North America and 2007-2010 NHANES participants who were used for comparison to the LTMDS users. NHANES subjects were classified as non-dietary supplement (NS) users, single supplement/single purpose supplement (SS) users, multivitamin/mineral supplement (MVMS) users, and multiple dietary supplement (MDS) users. Supplement groups were compared for total, HDL and LDL cholesterol; triglycerides; glucose; insulin; CRP and $\mathrm{HbA1c} \geq 6.5 \%$, adjusting for age, sex, income, education, BMI, history of CVD, and medications for hypercholesterolemia and diabetes. Geometric mean (95\% confidence interval) LDL cholesterol was significantly lower ( $<<0.05)$ for all supplement groups (SS: 110 (104-117) mg/dL; MVMS: 113 (107-119) mg/dL; MDS: 115 (111-118) mg/dL; LTMDS: 112 (105, 119) mg/dL) compared with the NS users $(122(118-126) \mathrm{mg} / \mathrm{dL})$. Compared with the NS group, MDS users had significantly $(P<0.05)$ lower mean total cholesterol (198 (194-201) vs 201 (197, 206) mg/dL), MDS and LTMDS users higher mean HDL cholesterol (54.2 (53.3-55.1) and 60.0 (57.4, 62.7) $\mathrm{mg} / \mathrm{dL}$ vs 52.0 (50.8-53.3) mg/dL), LTMDS users lower fasting glucose (100 (98-103) vs 105 (103-106) mg/dL), SS and MDS users lower insulin (8.4 (7.4-9.6) and 9.1 (8.3-9.9) vs 10.2 (9.4-11.1) $\mu \mathrm{lU} / \mathrm{mL})$, and MDS users lower CRP (\% $\geq 1.5 \mathrm{mg} / \mathrm{L}, 50.8$ (47.9-53.6) vs 57.0 (52.4-61.6) $\%)$. These findings add to the evidence that use of dietary supplements may provide cardiometabolic health benefits.
\end{abstract}

Keywords: Dietary supplements, cardiometabolic risk, diabetes, HbA1c, NHANES

\section{Introduction}

Dietary supplement use is widespread in the US. Approximately one-half of all adults used a dietary supplement in the past 30 days, and trends in both traditional dietary supplements and botanical dietary supplements appear to be stable since 1999 [1, 2]. The five most commonly used dietary supplements were multivitamin and mineral supplements (MVMS) (32\%), calcium (12\%), omega-3 or fish oil (10\%), botanicals (8\%) and vitamin C (7\%) [3]. Prevalence of supplement use varies based on a wide range of participant characteristics $[1,3]$. The overall prevalence of dietary supplement use is higher in women, and increases with age, income and education, and is highest in non-Hispanic whites relative to other racial and ethnic groups. Use of supplements was also positively related to better health and lifestyle choices and self-reported health status. Respondents reported that $23 \%$ of supplements were taken based on the recommendation of a physician or other health care provider with MVMS, calcium, omega-3/fish oils and vitamin $\mathrm{D}$ being the most frequently recommended supplements [3].

Despite this widespread acceptance of supplements on the part of the public and health care providers, questions about both the safety and effectiveness of dietary supplements continue to be raised [4]. The gold standard for determining the safety and efficacy of a new pharmaceutical agent is the double-blind, placebo-controlled, randomized clinical trial (RCT), and there has been a movement to hold supplements to the same standard, but this is exceedingly difficult to do for a number of reasons $[5,6]$. Observational studies are an alternative to RCTs for considering the question of supplement safety and efficacy, although these studies can be plagued by many issues related to confounding by other healthy lifestyle behaviors associated with supplement use and measurement of usual supplement use. Block et al. [7] addressed the former issue by applying this observational approach to an unusual population whose nutritional practice included long-term use of multiple dietary supplements to study this question of 
supplement safety and efficacy. They focused on a community defined by their active engagement with the social marketing arm of a supplement company. From this population, Block et al. characterized the health status of a sample of 278 long-term (20+ years) multiple dietary supplement (LTMDS) users. When compared with NHANES controls who either took no supplements or took only a multivitamin, the LTMDS users had significantly better status for an array of nutrients and consistently demonstrated a lower cardiometabolic risk profile based on lipoprotein, C-reactive protein (CRP) and total homocysteine concentrations. They also had a significantly lower prevalence of diabetes.

In the present study, designed as a follow-up to the earlier study of Block et al. [7], we examined cardiometabolic risk markers including diabetes markers and medication use to further explore the finding of Block and colleagues after an additional 10 years of multiple dietary supplement use to determine if cardiometabolic health benefits associated with the long-term multiple dietary supplement (LTMDS) use persisted over time and to see if these benefits were unique to LTMDS use or were also observed among shorter-term multiple dietary supplement users and single supplement users.

\section{Subjects and methods}

\section{Study participants}

Study participants were obtained from two sources, a study of the demographic characteristics and the cardiometabolic health status of LTMDS users from the United States and Canada conducted by a dietary supplement manufacturer and distributor (Shaklee Corporation), and participants from the 2007-2010 National Health and Examination Survey (NHANES), who were used for comparison to the LTMDS user sample for cardiometabolic risk factors as these were the most recent survey years with all the comparable biomarkers. The study was conducted in accordance with the Declaration of Helsinki. All study data collection procedures and protocols for the LTMDS cohort were approved by the Hummingbird Institutional Review Board (protocol \#2015-23). Protocols and procedures for analyses of blood samples and data analyses performed at Tufts University were approved by the Tufts Health Science Campus Institutional review Board (protocol \#11868). All study procedures and protocols for the NHANES surveys were approved by the National Center for Health Statistics (NCHS) Research Ethics Review Board (protocol \#2005-06)

Long-term multiple dietary supplement (LTMDS) users were Shaklee distributors and members who participated in an earlier study in which Block et al. [7] characterized the health status of 278 individuals who had used multiple dietary supplements for at least 20 years in the original study. At the time of recruitment into the present study, participants had used multiple dietary supplements for 30 years or more. Participants from this cohort who were planning to attend the 2015 annual convention for Shaklee distributors and members received an email from Shaklee Corporation inviting them to participate in the study at the convention site. Cohort participants who did not plan to attend the convention were contacted to schedule home visits. All participants received a follow-up email from Shaklee Corporation that included an explanation of the study, a consent form that included the Medical Research Subject's Bill of Rights, and a telephone number to contact Shaklee Health Sciences staff in the event individuals had questions pertaining to participating in the study or giving consent. The email also included a link to online questionnaires pertaining to diet, physical activity, demographic information, health status, medical history, and use of Shaklee foods and dietary supplements, which participants were only able to access after having acknowledged reading the consent form and having checked a box indicating their consent to participate in the study.

Subjects were emailed details of their laboratory appointment including instructions for fasting. Subjects were instructed to fast from dietary supplements, food, and beverages other than water and plain coffee or tea, for 12 hours prior to their clinical appointment. A written informed consent for the clinical study and all data and blood samples was obtained by personnel from Midwest Biomedical Research (Chicago, IL). Trained personnel measured height, weight, and abdominal circumference, and obtained a fasting blood sample (approximately $30 \mathrm{~mL}$ ).

Of the original 278 LTMDS use cohort participants, 191 members participated at the annual convention and 44 had home visits. Of the 235 subjects enrolled into the follow-up study, 204 subjects provided a blood specimen for laboratory analysis; only 195 subjects had complete laboratory results because some late remote blood samples were not available for complete analysis prior to the study end date. After excluding subjects with missing clinical and questionnaire data, there were 176 subjects with complete data on all questionnaires, clinical exam and laboratory results. Additional LTMDS users were excluded from analysis if they had a history of cancer other than nonmelanoma skin cancer $(n=17)$, were pregnant or lactating $(\mathrm{n}=1)$, or were children when they started supplements 30 years ago $(\mathrm{n}=2)$. The final analytic sample included $\mathrm{n}=156$ subjects.

Participants from the 2007-2010 NHANES were used for comparison to the LTMDS sample. NHANES is a nationally represented study of health and nutritional status of adults and children in the United States, with continuous data collection in two-year cycles starting in 1999 [8]. Each 
NHANES cycle included an initial in-person home interview and a subsequent study visit at a specially-designed and equipped Mobile Examination Center (MEC). To match the characteristics of the LTMDS sample, the 20,868 subjects available from 2007-10 NHANES surveys were restricted to 2,310 non-Hispanic white (NHW) adults, $40+$ years of age, who did not currently smoke, were not pregnant and were free of cancer. The selection of $40+$ years as a comparison group was based on the sampling design of NHANES and the availability of morning fasting subsample data collected in participants $40+$ years of age.

\section{Outcomes and laboratory methods}

The outcomes included total cholesterol (plasma), HDL cholesterol (plasma) and LDL cholesterol (calculated), triglycerides (plasma), glucose (serum for LTMDS and plasma for NHANES), insulin (serum), and high sensitivity CRP (plasma), and the prevalence of elevated hemoglobin A1c (HbA1c) and diabetes. For LTMDS and NHANES participants, elevated $\mathrm{HbA1c}$ was defined as $\mathrm{HbA1c} \geq 6.5 \%$, and diabetes was defined as having any of the following: 1) elevated HbA1c, 2) self-report of current diabetes medication use, or 3) self-report of a diagnosis of diabetes by a physician.

For the LTMDS participants, a certified phlebotomist drew approximately $30 \mathrm{ml}$ of blood on site during Cleveland convention or at subject's home. Blood samples, except those for whole blood, were centrifuged. Samples collected in Cleveland were stored immediately at $-20^{\circ} \mathrm{C}$ and shipped to MB Clinical Research (Boca Raton, FL) where they were stored at $-80^{\circ} \mathrm{C}$ until further analysis. The samples collected in people's homes were shipped with cold packs (not frozen) to the MB Clinical Research and were transferred to $-80^{\circ} \mathrm{C}$ storage upon receipt.

Lipoproteins were measured in EDTA plasma on a clinical chemistry analyzer (AU480 Clinical Chemistry Analyzer, Beckman Coulter, Inc., Brea CA). Cholesterol was measured by an enzymatic procedure $[9,10]$ as specified in the Olympus AU480 Procedural Insert (Cholesterol OSR6116 and OSR6216). The intra- and inter-assay coefficient of variation (CV) were $2.0 \%$ and $2.8 \%$ respectively. Triglycerides were measured by a series of coupled enzymatic reactions [11, 12] and as specified in the Olympus AU400 Procedural Insert (Triglycerides OSR6033 and OSR6133). The intra- and inter-assay CVs were $2.0 \%$ and $3.4 \%$ respectively. HDL cholesterol was measured by a two-phase reaction with colorimetric endpoint detection as specified in the Olympus AU480 Procedural Insert (HDL-Cholesterol OSR6187 and OSR6287). The intraand inter-assay CVs were $3.0 \%$ and $5.0 \%$ respectively. The Friedewald formula [13] was used to estimate LDL cholesterol. Glucose was measured in serum on a clinical chemistry analyzer, (AU480 Clinical Chemistry Analyzer, Beckman Coulter, Inc., Brea CA) and as specified in the Olympus AU480 Procedural Insert for glucose. The intraand inter-assay CVs were $2.0 \%$ and $3.4 \%$, respectively. HbA1c levels were measured using the turbidimetric inhibition immunoassay (Tina-quant ${ }^{\circledR}$, RocheDiagnostics, Mannheim, Germany). High sensitivity CRP and insulin were measured in serum by solid-phase, two-site chemiluminescent immunometric assays using the IMMULITE 2000, (Siemens Healthcare Diagnostics, Los Angeles, CA) and according to Babson [14]. The intra- and inter-assay CVs were $3.0 \%$ and $5.0 \%$ respectively for hsCRP and $5.8 \%$ and $7.0 \%$ for insulin.

For the NHANES participants, total and HDL cholesterol and triglycerides were analyzed using a Roche Modular $\mathrm{P}$ chemistry analyzer (Roche Diagnostics, Indianapolis, IN). Total and HDL cholesterol were analyzed regardless of fasting status while triglycerides measurements were restricted to the fasting morning sample of MEC participants. Serum LDL cholesterol was calculated for those seen in morning session only using the Friedewald calculation [13]. HbA1c measurements were performed on the A1c G7 HPLC Glycohemoglobin Analyzer. Glucose measurements were performed on the Roche Modular P chemistry analyzer (Roche Diagnostics, Indianapolis, IN) using the hexokinase assay. Fasting insulin was analyzed using Merocodia Insulin ELISA kits. All analyses for glucose and insulin were restricted to the MEC fasting morning sample. CRP was analyzed using a Behring Nephelometer for quantitative determination.

\section{Supplement assessment}

The LTMDS participants answered an online medical and supplement history questionnaire (MHQ) developed by NutritionQuest (Berkeley, CA), indicating the frequency and amount of dietary supplements they were consuming. For NHANES participants, dietary supplement use was assessed at the home visit using the Dietary Supplement and Prescription Medication Section of the Sample Person Questionnaire. This questionnaire collects self-report data on supplement use during the previous 30 days. To be more consistent with the MHQ supplement question regarding weekly use of dietary supplements for the LTMDS participants, we required the NHANES participants to report consumption at least 4 times in the last 30 days.

NHANES participants were classified into four supplement groups: those reporting no dietary supplement (NS) use, multivitamin/mineral supplement (MVMS) users (defined as a single supplement with $\geq 10$ multivitamin or minerals), single supplement/single purpose supplement (SS) users (defined as a single supplement containing 1-9 vitamins or minerals), and those subjects taking $\geq 2$ supplement pills per day (MDS users). 


\section{Medication use}

In the online MHQ LTMDS participants were asked to list all medications they were taking with specific questions related to diabetes medication. NHANES participants were asked if they have taken prescription medications in the past month [15]. Those who answer "yes" were asked to show the container of the medications used and the interviewer recorded the drug name and matched it to an existing drug in the Multum Lexicon Plus ${ }^{\circledR}$ database [16]. Each drug was further classified into therapeutic and ingredient levels using the database. We utilized the top tier classification in which all drugs consumed fall into one of the 17 therapeutic categories. The LTMDS medication responses were grouped into the same 17 categories for direct comparison. Medications classified as hormone/hormone modifiers or nutritional products were removed from all analyses. Any category with less than $10 \%$ response was reclassified into Miscellaneous Agents for both NHANES and LTMDS participants, resulting in eight categories. The numbers of medications were then summed across all drug categories to obtain a total number of medications recorded per subject.

\section{Covariates}

Covariates included age, sex, income $(<\$ 40,000$, $\geq \$ 40,000$, undisclosed), education level ( $\leq 8$ th grade, 9-11th grade, high school, some college, and college graduate and above), body mass index (BMI, $\mathrm{kg} / \mathrm{m}^{2}$ ), history of cardiovascular disease (CVD), dietary fiber (g/day) and energy (kcal/day) intakes, and lipid lowering and diabetes medication use.

LTMDS participants completed electronic questionnaires pertaining to demographic information, physical activity, health status, and medical history developed by NutritionQuest (Berkeley, CA). Weight, height and waist circumference were measured at the clinical visit by trained personnel. Diet was assessed in the LTMDS participants using the 2014 Block FFQ (NutritionQuest, Berkeley, CA). Data from this questionnaire was then analyzed by NutritionQuest and specific nutrient and food group summaries were derived.

For NHANES participants, information on demographics, health status, and medical history were obtained by trained interviewers at the home and MEC visits, and height and weight were measured by trained personnel at the MEC exam. NHANES participants' dietary intakes were assessed with a 24-hour recall using the USDA's dietary data collection instrument, the Automated Multiple Pass Method. Subjects were administered the initial recall in person. A second recall was performed via telephone 3-10 days later. Nutrient data for this analysis was derived from the MEC (day 1) recall only.

\section{Statistical analysis}

LTMDS and NHANES data were combined for analysis. In order to preserve complex sampling design of NHANES, we incorporated the strata and primary sampling unit (PSU) designations for NHANES participants and recalibrated the sample weights as the original sample weight divided by the sum of the sample weights within the supplement category. The LTMDS sample was assigned one strata, and each LTMDS subject was given a unique PSU and a weight of $1 /$ LTMDS sample size. This recalibration allowed for equal weighting for each supplement group and more precise variance estimation but no longer provides estimates that are considered nationally representative. We used SUDAAN statistical software (RTI, Research Triangle Park, North Carolina, USA) and SAS survey procedures (SAS, Cary, North Carolina, USA) for all analyses to account for the complex sampling design in the variance estimates.

Participant characteristics were evaluated across five supplement groups: NHANES NS, SS, MVMS and MDS users, and LTMDS users. Categorical data are presented as percentages and compared using chi-square and logistic regression analyses. Continuous measures are presented as least square means (LSMEANS) and compared by multiple regression. Body composition and dietary variables were adjusted for age and sex. Dietary fiber was additionally adjusted for energy. Logarithmic transformations were applied to highly skewed outcomes and geometric means presented.

The relationships between the outcomes and supplement use category were examined using analysis of covariance (ANCOVA). Supplement use categories included the LTMDS users and NHANES MDS, SS, and MVMS users and those reporting no dietary supplement (NS) use. Statistical comparisons were performed between the NS use category and the four supplement user categories. We also compared the LTMDS and NHANES MDS categories as these groups were the most similar in terms of supplement use behavior. To address potential confounding, we adjusted the main model for the following items: age, sex, income, education level, BMI and history of CVD. A second model had additional adjustment for dietary fiber intake and energy intake. Cholesterol outcomes were additionally adjusted for lipid lowering medication use and glucose and insulin for diabetes medication use. For HbA1c, we also tested for interactions between diabetes medication use and supplement group using the same ANCOVA model and based on the Wald statistic. Logarithmic and square root transformations were applied to skewed outcomes and results were transformed back to their original scale for presentation. P values of $<0.05$ were considered statistically significant. 


\section{Results}

\section{Participant characteristics}

Study participants in these analyses included 156 LTMDS users who were combined with 2,310 NHANES 2007-10 participants. Many demographic and health-related characteristics were associated with dietary supplement use among the NHANES participants (table 1). NHANES SS and MDS users were older and more likely to be female than the NS group, whereas MVMS users had higher average income. The NS group had a significantly higher percent of participants with less than a $9^{\text {th }}$ grade education than SS, MVMS, and MDS supplement users. The MVMS and MDS users were more likely to take cholesterol-lowering medication, MDS users less likely to take diabetes medications, SS and MDS users to have lower BMI, and MDS users to consume more dietary fiber than the NS group. MVMS and MDS also reported higher energy intakes compared with the NS group.

The LTMDS study participants differ from the nationally representative sample of NHANES 2007-10 NS users on every demographic and health-related characteristic we considered, except energy intake (table 1). In spite of limiting the age and income range of the NHANES participants to achieve a closer match with the LTMDS cohort, the LTMDS participants were more likely to be older and have higher incomes than the NHANES NS users. They also were more likely to be women, more educated, and less likely to be taking medications for diabetes, hypertension or elevated cholesterol, and they had lower BMI and higher dietary fiber intakes. Similar differences were observed between the LTMDS users and NHANES MDS users, except that the proportion of women in LTMDS and MDS user groups was not different and the LTMDS users also reported a lower energy intake than the MDS users.

\section{Supplement use and cardiometabolic risk factors}

None of the supplement groups had a significantly different prevalence of diabetes relative to the NS group (table 2). However, the percent of people with the elevated HbA1c $(\geq 6.5 \%)$ was approximately $50 \%$ lower in LTMDS users than the NS use group $(\mathrm{P}<0.006)$. The SS users also had a significantly lower percent of people with the elevated HbA1c than the NS group $(\mathrm{P}<0.02)$. Although we failed to detect a significant interaction between diabetes medication use and supplement use categories, we stratified these analyses by diabetes medication use because of the extreme differences in prevalence of elevated $\mathrm{HbA1c}$ between subjects taking and not taking diabetes medication. After stratification, we observed that the associations between supplement use and elevated HbA1c seen for the overall study sample were present only among the LTMDS and SS users not taking diabetes medication.

Many cardiometabolic biomarkers were beneficially associated with supplement use (table 3). All of the vitamin supplement use categories demonstrated significantly lower LDL cholesterol compared with the NS users $(\mathrm{P}<$ 0.05). The MDS users had lower total cholesterol levels ( $P$ $=0.05)$ compared with the NS user group, whereas the LTMDS $(\mathrm{P}<0.001)$ and MDS $(\mathrm{P}=0.02)$ users had higher HDL cholesterol relative to the NS group. LTMDS users had a lower fasting glucose $(\mathrm{P}<0.009)$, the MDS ( $\mathrm{P}=$ $0.02)$ and SS $(\mathrm{P}=0.01)$ groups had lower insulin, and the MDS group $(\mathrm{P}=0.02)$ had lower $\mathrm{CRP}$ levels compared with the NS group. LTMDS users also demonstrated significantly higher HDL cholesterol and significantly lower glucose concentrations than the MDS users.

\section{Supplement use and prevalence of medication use}

We considered the association between supplement use and polypharmacy across eight medication categories (table 4). For almost all medication categories, the LTMDS group had a much lower prevalence of medication use than all NHANES supplement groups. The median $\left(25^{\text {th }}\right.$ and $75^{\text {th }}$ percentiles) were $1.98(0.69,3.83)$ for the NS users, 2.50 $(1.11,3.88)$ for the SS users, $2.08(0.95,3.65)$ for the MVMS users, $2.20(1.00,3.96)$ for the MDS users and $0.00(0.00$, 0.30 ) for the LTMDS users.

\section{Discussion}

This study was designed to examine if the potential cardiometabolic benefits associated with the long-term use of multiple dietary supplements seen in the original LTMDS study by Block et al. [7], based on 20 or more years of multiple supplement use, persisted over time. The original study observed that the LTMDS users had a lower total cholesterol, triglycerides and CRP levels and higher HDL cholesterol than the NHANES NS group. Ten years later, after 30 or more years of MDS use, we again observed that the LTMDS users had better cardiometabolic profiles relative to a similarly aged nationally representative sample of non-supplement users from the 2007-2010 NHANES.

Both our follow-up study and Block and colleagues' original study [7] observed that LTMDS users had a higher HDL cholesterol levels relative to the NHANES NS users. However, there were also differences in the observed associations with other cardiometabolic risk factors over time. 
Table 1. Participant characteristics: weighted mean or percent (95\% confidence intervals)

\begin{tabular}{|c|c|c|c|c|c|}
\hline & \multicolumn{4}{|c|}{ NHANES 2007-10 } & \multirow[b]{2}{*}{ LTMDS $^{1}$} \\
\hline & $\begin{array}{c}\text { No supplement } \\
\text { use }\end{array}$ & $\begin{array}{l}\text { Single supplement/ } \\
\text { single purpose } \\
\text { supplement }\end{array}$ & $\begin{array}{l}\text { Multi-vitamin/ } \\
\text { mineral } \\
\text { supplement }\end{array}$ & $\mathrm{MDS}^{1}$ & \\
\hline Sample N & 866 & 199 & 298 & 947 & 156 \\
\hline Age $(\text { years) })^{2}$ & $55.0(54.0,56.0)$ & $58.9(57.1,60.6)^{3}$ & $54.3(52.9,55.8)$ & $60.4(59.6,61.2)^{3}$ & $71.9(70.7,73.1)^{3,4}$ \\
\hline Female (\%) & $42.8(39.5,46.1)$ & $59.3(50.9,67.8)^{3}$ & $46.1(38.9,53.3)$ & $65(61.4,68.6)^{3}$ & $60.9(53.2,68.6)^{3}$ \\
\hline Hispanic (\%) & 0 & 0 & 0 & 0 & $1.8(0.69,4.8)$ \\
\hline \multicolumn{6}{|l|}{ Income (\%) } \\
\hline$<\$ 40.000$ & $32.7(26.5,38.8)$ & $31.3(23.9,38.8)$ & $22.7(16.7,28.7)^{3}$ & $33.1(28.9,37.2)$ & $6.4(2.5,10.3)^{3,4}$ \\
\hline$>\$ 40,000$ & $61.8(55.6,68.0)$ & $66.6(59.6,73.6)$ & $70.3(63.4,77.2)^{3}$ & $61.7(57.9,65.4)$ & $78.2(71.7,84.7)^{3,4}$ \\
\hline Refused & $5.6(4.1,7.1)$ & $2.1(0.2,4.0)^{3}$ & $7.0(3.2,10.7)$ & $5.3(3.3,7.2)$ & $15.4(9.7,21.1)^{3,4}$ \\
\hline \multicolumn{6}{|c|}{ Highest level of education (\%) } \\
\hline Less than 9th grade & $4.4(2.4,6.4)$ & $2.3(0.9,3.6)^{3}$ & $2.5(0.7,4.3)^{3}$ & $2.2(1.0,3.5)^{3}$ & $0(. .,)^{3,4}$ \\
\hline 9-11th grade & $9.6(7.0,12.2)$ & $11.0(5.3,16.7)$ & $5.5(3.3,7.7)^{3}$ & $8.4(6.2,10.5)$ & $1.9(0.0,4.1)^{3,4}$ \\
\hline High school graduate & $25.2(22.0,28.4)$ & $24.2(15.9,32.5)$ & $23.6(16.9,30.2)$ & $25.1(22.1,28.2)$ & $10.9(6.0,15.8)^{3,4}$ \\
\hline Some college & $28.8(25.1,32.5)$ & $25.4(19.0,31.8)$ & $29.6(22.8,36.3)$ & $27.8(24.5,31.1)$ & $28.8(21.7,36.0)$ \\
\hline College and above & $32.0(27.5,36.5)$ & $37.1(27.4,46.9)$ & $38.9(29.4,48.3)$ & $36.5(32.1,40.9)$ & $58.3(50.5,66.1)^{3,4}$ \\
\hline \multicolumn{6}{|l|}{ Medication use } \\
\hline Diabetes $(\%)^{5}$ & $9.1(6.3,11.8)$ & $8.5(4.7,12.3)$ & $8.6(4.5,12.7)$ & $5.6(3.7,7.5)^{3}$ & $1.0(0.0,3.9)^{3,4}$ \\
\hline Blood Pressure $(\%)^{5}$ & $40.2(36.1,44.3)$ & $42.3(35.3,49.3)$ & $45(36.9,53.1)$ & $38.6(35.4,41.7)$ & $0.0(0.0,5.4)^{3,4}$ \\
\hline Cholesterol $(\%)^{5}$ & $39(34.7,43.3)$ & $46.8(38.4,55.1)$ & $45.9(40.8,51.1)^{3}$ & $52.6(48.9,56.4)^{3}$ & $1.1(0.0,5.4)^{3,4}$ \\
\hline CVD $(\%)^{5}$ & $16.8(14.2,19.4)$ & $15.5(11.1,19.8)$ & $14.2(10.9,17.5)$ & $14.0(11.2,16.8)$ & $5.3(0.3,10.3)^{3,4}$ \\
\hline $\mathrm{BMI}\left(\mathrm{kg} / \mathrm{m}^{2}\right)^{6}$ & $29.6(29.0,30.1)$ & $28.5(27.7,29.4)^{3}$ & $29.0(28.3,29.6)$ & $27.7(27.2,28.1)^{3}$ & $25.9(25.2,26.7)^{3,4}$ \\
\hline Fiber intake (g/day) $)^{7,8}$ & $14.7(14,15.5)$ & $16(14.7,17.5)$ & $15.1(14.2,16.1)$ & $16.9(16.2,17.7)^{3}$ & $19.4(18.3,20.6)^{3,4}$ \\
\hline Energy (kcal/day $)^{6,8}$ & $1799(1735,1864)$ & $1863(1754,1978)$ & $1937(1868,200)^{3}$ & $1930(1870,1993)^{3}$ & $1739(1644,1840)^{4}$ \\
\hline
\end{tabular}

${ }^{1} \mathrm{MDS}=$ multiple dietary supplement; LTMDS = long-term multiple dietary supplement. ${ }^{2}$ NHANES participants aged years 80 and over were top-coded at 80 years. ${ }^{3}$ Significantly different from the "No supplement use" category $(p<0.05) .{ }^{4}$ LTMDS significantly different from MDS ( $\left.p<0.05\right) .{ }^{5}$ Age and sex adjusted means and $95 \% \mathrm{Cl}$ presented. ${ }^{6} \mathrm{Age}$ and sex adjusted geometric means and $95 \% \mathrm{Cls}$ presented. ${ }^{7}$ Age, sex and energy adjusted geometric means and $95 \% \mathrm{Cls}$ presented. ${ }^{8}$ Dietary data derived from the Block FFQ (2014, version 3) for LTMDS participants and 24-hour recall for NHANES participants.

Total cholesterol was no longer significantly lower in the LTMDS users in this follow-up although the basis for this seems to be a lower mean total cholesterol in the NHANES 2007-10 NS users $(201 \mathrm{mg} / \mathrm{dL})$ than in the NHANES 20012002 and NHANES III NS users $(212 \mathrm{mg} / \mathrm{dL})$, who served as the comparison group in the original Block et al. [7] study. Triglycerides were also not significantly lower in the LTMDS users relative to the NS users in the present study, as had been seen in the original study, but as with the cholesterol levels, the circulating triglyceride concentrations in the NHANES 2007-10 NS users ( $109 \mathrm{mg} / \mathrm{dL}$ ) were substantially lower than reported by Block et al. in the NHANES 2001-2002 and NHANES III NS users (180 mg/dL). We also did not replicate the lower CRP concentrations in the LTMDS users seen by Block et al. but we could not directly compare findings of the two studies as we used a cutoff of 1.5 $\mathrm{mg} / \mathrm{L}$ to define elevated CRP due to the highly skewed nature of this biomarker whereas Block and colleagues used the mean to characterize CRP levels.

We observed a significant beneficial association between LTMDS use and LDL cholesterol, which Block et al. [7] did not observe, and a beneficial association between fasting glucose concentrations and LTMDS use, which Block and colleagues did not consider in the original study. We also did not observe a significantly lower prevalence of diabetes relative to the NHANES NS users as previously seen in the original study of the LTMDS users, which may be due in part to a small number of LTMDS users and a resulting low statistical power.

We also observed better metabolic profiles for supplement users in the NHANES 2007-10 sample relative to the NS group. The NHANES MDS users had significantly higher HDL and lower LDL cholesterol, as well as lower total cholesterol, insulin, and CRP concentrations than NS users. The NHANES MVMS users had a significantly lower LDL cholesterol levels and the SS users had a significantly lower prevalence of elevated $\mathrm{HbA1c}$, and lower LDL cholesterol and insulin levels than the NHANES NS users.

Our findings suggest that dietary supplement use, particularly use of MDS, may provide cardiometabolic benefit, consistent with the earlier observations in the LTMDS cohort [7]. Our findings also support evidence from earlier observational studies on dietary supplement use and 
Table 2. Prevalence of diabetes and elevated HbA1c

\begin{tabular}{|c|c|c|c|c|c|}
\hline & \multicolumn{4}{|c|}{ NHANES 2007-10 } & \multirow[b]{2}{*}{$\begin{array}{l}\text { LTMDS }^{2} \\
(n=146)\end{array}$} \\
\hline & $\begin{array}{l}\text { No supplement } \\
\text { use }(n=862)^{1}\end{array}$ & $\begin{array}{c}\text { Single supplement/single } \\
\text { purpose supplement }(n=198)\end{array}$ & $\begin{array}{l}\text { Multi-vitamin/mineral } \\
\text { supplement }(n=297)\end{array}$ & $\begin{array}{c}\text { MDS }^{2} \\
(n=938)\end{array}$ & \\
\hline \multicolumn{6}{|c|}{ Diabetes prevalence $(\%)^{3}$} \\
\hline Model $1^{4}$ & $11.9(9.6,14.2)$ & $12.5(8.2,16.7)$ & $11.4(6.9,15.9)$ & $11.5(9.1,13.9)$ & $7.6(3.1,12.1)$ \\
\hline P-value ${ }^{5}$ & & 0.82 & 0.82 & 0.78 & 0.13 \\
\hline Model $2^{6}$ & $12.1(9.7,14.5)$ & $12.9(8.3,17.5)$ & $11.5(6.8,16.2)$ & $11.1(8.7,13.4)$ & $7.2(2.6,11.8)$ \\
\hline P-value ${ }^{5}$ & & 0.75 & 0.79 & 0.53 & 0.09 \\
\hline \multicolumn{6}{|c|}{ Prevalence of elevated $\mathrm{HbA} 1 \mathrm{c}(\%)^{7}$} \\
\hline Model $1^{8}$ & $9.3(7.7,10.9)$ & $5.6(3.3,7.9)$ & $9.3(6.7,11.8)$ & $8.2(6.8,9.5)$ & $4.7(2.0,7.5)^{9}$ \\
\hline P-value & & 0.02 & 0.97 & 0.32 & 0.006 \\
\hline Model $2^{8}$ & $9.4(7.7,11.0)$ & $5.7(3.3,8.1)$ & $9.3(6.7,11.9)$ & $7.8(6.5,9.2)$ & $4.7(2.0,7.4)^{9}$ \\
\hline P-value & & 0.03 & 0.97 & 0.18 & 0.005 \\
\hline \multicolumn{6}{|c|}{$\begin{array}{l}\text { Prevalence of elevated } \\
\mathrm{HbA} 1 \mathrm{c}(\%)^{7} \text { by diabetes } \\
\text { medication use Yes }(n=178)^{10}\end{array}$} \\
\hline Model 1 & $72.0(61.8,82.3)$ & $80.9(60.6,101.1)$ & $77.2(60.9,93.4)$ & $73.4(64.1,82.7)$ & $74.3(29.5,119.2)$ \\
\hline P-value & & 0.40 & 0.54 & 0.85 & 0.93 \\
\hline Model 2 & $69.3(60.3,78.4)$ & $77.9(62.5,93.3)$ & $79.8(64.1,95.6)$ & $72.6(63.2,82.0)$ & $85.7(37.4,134.0)$ \\
\hline P-value & & 0.31 & 0.22 & 0.62 & 0.51 \\
\hline \multicolumn{6}{|c|}{ No $(n=2263)^{11}$} \\
\hline Model 1 & $5.5(3.9,7.2)$ & $1.4(0.0,3.0)$ & $4.6(2.3,6.9)$ & $4.2(3.0,5.4)$ & $0.5(0.0,2.9)^{9}$ \\
\hline P-value & & 0.003 & 0.49 & 0.2 & 0.001 \\
\hline Model 2 & $5.6(3.8,7.3)$ & $1.4(0.0,3.2)$ & $4.4(2.2,6.7)$ & $4.0(2.9,5.1)$ & $0.5(0.0,2.7)^{9}$ \\
\hline P-value & & 0.005 & 0.40 & 0.15 & 0.001 \\
\hline
\end{tabular}

${ }^{1} \mathrm{HbA1c}$ data were missing in all NHANES and LTMDS supplement use categories. ${ }^{2} \mathrm{MDS}=$ multiple dietary supplement use; LTMDS = long-term multiple dietary supplement use. ${ }^{3}$ Diabetes prevalence defined as $\mathrm{HbA} 1 \mathrm{c} \geq 6.5$, self-report of diabetes or diabetes medication use. ${ }^{4}$ Model 1 : adjusted for age, sex, income $(\langle \$ 0,000, \geq \$ 40,000$, undisclosed), education level ( $\leq 8$ th grade, $9-11$ th grade, high school, some college, and college and above), BMI and history of CVD. ${ }^{5}$-value for comparison to the "no supplement use" category. ${ }^{6}$ Model 2: Model 1 with additional adjustment for dietary fiber intake and energy intake. ${ }^{7}$ Elevated $\mathrm{HbA} 1 \mathrm{c}$ defined as $\geq 6.5 \% .{ }^{8}$ Includes covariates as described for diabetes models with additional adjustment for diabetes medication use (y/n). ${ }^{9}$ LTMDS category significantly different from NHANES MDS category $(\mathrm{P}<0.05) .{ }^{10} \mathrm{n}$ per supplement use category: no supplement use, 82 ; single supplement/ single purpose supplement 18; multi-vitamin/mineral supplement 24; MDS 50; LTMDS $4 .{ }^{11} \mathrm{n}$ per supplement use category: no supplement use, 780; single supplement/ single purpose supplement 180; multi-vitamin/mineral supplement 273; MDS 888; LTMDS 142.

cardiometabolic risk [17-22], although it is beyond the scope of this paper to compare our present findings against all of the evidence for and against a role of dietary supplement use in cardiometabolic risk. The literature is too extensive to review here given the large number of ingredients found in dietary supplements included in this work and the variety of cardiometabolic risk markers considered.

There remain many who question health benefits of dietary supplements apart from prevention of vitamin and mineral deficiencies, based largely on the failure to demonstrate efficacy in RCTs and the argument that the efficacy of dietary supplements in disease prevention can only be addressed using RCTs $[4,23,24]$. While RCTs are the gold standard for scientific evidence for treatment of disease and for determining the safety and efficacy of pharmaceutical agents and other medical treatments, the use of RCTs to study the role of dietary supplements in disease prevention, particularly for active ingredients that are commonly consumed through foods, is much more difficult than for pharmaceuticals for many reasons $[5,6]$. One critical issue is the ubiquitous exposure to many components of dietary supplements. Unlike pharmaceutical agents, most individuals are exposed to the nutrients or other ingredients found in dietary supplement on a daily basis through their diet so identifying a truly unexposed placebo group is usually difficult at best. Because of contamination of the placebo group, RCTs of supplements can end up as a comparison of supraphysiological doses of nutrient intakes or placebo groups with physiological intakes and/or adequate nutrient status [25-27].

However, we also recognize, as seen in the present study, that there are many health and lifestyle differences between individuals who use and do not use dietary supplements. Therefore, evidence from observational studies alone is insufficient by itself to demonstrate health benefits of dietary supplements. Consequently, in place of long-term RCTs of dietary supplements and disease outcomes, we may need to rely on RCT evidence that dietary supplements can affect intermediate risk factors. Such evidence from RCTs of risk factors could then be coupled with evidence from 
Table 3. Cardiometabolic risk biomarkers: adjusted least square means and percentages ( $95 \%$ confidence intervals) by supplement group

\begin{tabular}{|c|c|c|c|c|c|}
\hline \multirow[b]{2}{*}{ Outcome } & \multicolumn{4}{|c|}{ NHANES 2007-10 } & \multirow[b]{2}{*}{$\begin{array}{l}\text { LTMDS }^{1} \\
(n=153)\end{array}$} \\
\hline & $\begin{array}{l}\text { No supplement } \\
\text { use }(n=861)\end{array}$ & $\begin{array}{l}\text { Single supplement } \\
\text { or single } \\
\text { purpose } \\
\text { supplement } \\
(n=197)\end{array}$ & $\begin{array}{l}\text { Multi-vitamin/mineral } \\
\text { supplement } \\
(n=295)\end{array}$ & $\begin{array}{c}M^{1} S^{1} \\
(n=936)\end{array}$ & \\
\hline \multicolumn{6}{|c|}{ Total Cholesterol $(\mathrm{mg} / \mathrm{dL})^{2}$} \\
\hline Model $1^{3}$ & $201(197,206)$ & $197(192,202)$ & $195(189,200)$ & $198(194,201)$ & $200(193,208)$ \\
\hline P-value ${ }^{4}$ & & 0.18 & 0.09 & 0.050 & 0.780 \\
\hline Model $2^{5}$ & $202(198,206)$ & $196(191,202)$ & $195(189,200)$ & $197(194,201)$ & $200(193,208)$ \\
\hline P-value & & 0.09 & 0.07 & 0.02 & 0.69 \\
\hline \multicolumn{6}{|c|}{ HDL-Cholesterol $(\mathrm{mg} / \mathrm{dL})^{2}$} \\
\hline Model 1 & $52.0(50.8,53.3)$ & $52.3(50.3,54.4)$ & $53.1(51.6,54.7)$ & $54.2(53.3,55.1)$ & $60.0(57.4,62.7)^{6}$ \\
\hline P-value & & 0.82 & 0.12 & 0.02 & $<0.001$ \\
\hline Model 2 & $52.2(50.9,53.4)$ & $52.3(50.3,54.5)$ & $53.2(51.6,54.9)$ & $54.2(53.3,55.0)$ & $60.1(57.5,62.8)^{6}$ \\
\hline P-value & & 0.86 & 0.11 & 0.02 & $<0.001$ \\
\hline \multicolumn{6}{|c|}{ Calculated LDL-Cholesterol (mg/dL) } \\
\hline Model 1 & $122(118,126)$ & $110(104,117)$ & $113(107,119)$ & $115(111,118)$ & $112(105,119)$ \\
\hline P-value & & 0.001 & 0.05 & $<0.001$ & 0.03 \\
\hline Model 2 & $122(118,127)$ & $110(104,117)$ & $113(107,120)$ & $115(111,118)$ & $112(105,119)$ \\
\hline P-value & & 0.001 & 0.05 & $<0.001$ & 0.03 \\
\hline \multicolumn{6}{|c|}{ Triglycerides $(\mathrm{mg} / \mathrm{dL})^{2,8}$} \\
\hline Model 1 & $109(104,114)$ & $113(103,123)$ & $118(107,130)$ & $106(100,112)$ & $101(92,111)$ \\
\hline P-value & & 0.53 & 0.13 & 0.56 & 0.23 \\
\hline Model 2 & $109(104,114)$ & $112(103,123)$ & $117(106,129)$ & $107(101,113)$ & $103(94,112)$ \\
\hline P-value & & 0.59 & 0.189 & 0.64 & 0.30 \\
\hline \multicolumn{6}{|c|}{ Fasting Glucose $(\mathrm{mg} / \mathrm{dL})^{2,8}$} \\
\hline Model 1 & $105(103,106)$ & $104(100,108)$ & $106(104,109)$ & $105(103,107)$ & $100(98,103)^{6}$ \\
\hline P-value & & 0.64 & 0.26 & 0.78 & 0.009 \\
\hline Model 2 & $105(103,106)$ & $104(100,108)$ & $106(103,109)$ & $105(103,107)$ & $101(98,103)^{6}$ \\
\hline P-value & & 0.71 & 0.35 & 0.88 & 0.03 \\
\hline \multicolumn{6}{|c|}{ Insulin $(\mu \mid U / m L)^{2,8}$} \\
\hline Model 1 & $10.2(9.4,11.1)$ & $8.4(7.4,9.6)$ & $10.8(9.9,11.7)$ & $9.1(8.3,9.9)$ & $9.2(8.1,10.3)$ \\
\hline P-value & & 0.01 & 0.39 & 0.02 & 0.17 \\
\hline Model 2 & $10.3(9.4,11.2)$ & $8.4(7.4,9.6)$ & $10.7(9.9,11.6)$ & $9.1(8.4,9.9)$ & $9.3(8.3,10.5)$ \\
\hline P-value & & 0.009 & 0.51 & 0.02 & 0.24 \\
\hline \multicolumn{6}{|c|}{ C-reactive protein $\geq 1.5 \mathrm{mg} / \mathrm{L}(\%)$} \\
\hline Model 1 & $57.0(52.4,61.6)$ & $53.4(46.5,60.3)$ & $54.9(49.7,60.1)$ & $50.8(47.9,53.6)$ & $48.4(39.2,57.6)$ \\
\hline P-value & & 0.47 & 0.56 & 0.02 & 0.15 \\
\hline Model 2 & $56.3(51.8,60.7)$ & $52.5(45.9,59.2)$ & $54.3(49.2,59.5)$ & $51.4(48.3,54.4)$ & $48.4(39.3,57.4)$ \\
\hline P-value & & 0.41 & 0.59 & 0.05 & 0.17 \\
\hline
\end{tabular}

${ }^{1} \mathrm{MDS}=$ multiple dietary supplement use; LTMDS = long-term multiple dietary supplement use. ${ }^{2}$ Geometric means and $95 \%$ Cls are presented. ${ }^{3}$ Model $1:$ adjusted for age, sex, income $(\langle \$ 40,000, \geq \$ 40,000$, undisclosed), education level ( $\leq 8$ th grade, $9-11$ th grade, high school, some college, and college and above), BMI and history of CVD. Cholesterol outcomes have additional adjustment for lipid lowering medication use and glucose and insulin outcomes have additional adjustment for diabetes medication use. ${ }^{4} \mathrm{P}$-value for comparison to the "no supplement use" category. ${ }^{5}$ Model 2: Model 1 with additional adjustment for dietary fiber intake and energy intake. ${ }^{6}$ LTMDS category significantly different from NHANES MDS category at the 0.05 alpha level. ${ }^{7}$ Data analyzed using square root transformation and back transformed to origorning subsample. ${ }^{8}$ Sample sizes for NHANES groups are as follows: No supplement use $n=387$, single supplement/single purpose $n=98$, multivitamin $n=143, \geq 2$ supplements per day $n=430$.

longitudinal observational studies of these risk factors and disease and RCTs of pharmacological agents demonstrating that modification of these same risk factors can affect risk of disease outcomes.
As with all studies, the design of the present study has both strengths and limitations. A major strength of the current study is the unique LTMDS study sample, a welldefined and well-documented sample of long-term users 
Table 4. Number (and percent) of medication use by supplement category

\begin{tabular}{lccccc}
\hline & \multicolumn{5}{c}{ NHANES 2007-10 } \\
\cline { 2 - 5 } Medication type & $\begin{array}{c}\text { No supplement } \\
\text { use }(\mathrm{n}=609)\end{array}$ & $\begin{array}{c}\text { Single supplement } \\
\text { or single purpose } \\
\text { supplement }(\mathrm{n}=158)\end{array}$ & $\begin{array}{c}\text { Multi-vitamin/mineral } \\
\text { supplement }(\mathrm{n}=224)\end{array}$ & $\begin{array}{l}\text { MDS }^{1} \\
(\mathrm{n}=786)\end{array}$ & $\begin{array}{l}\text { LTMDS }^{1} \\
(\mathrm{n}=156)\end{array}$ \\
\hline Cardiovascular & $383(36.9)$ & $105(41.8)$ & $144(41.0)$ & $492(45.3)$ & $22(14.1)$ \\
CNS Agents & $206(19.9)$ & $62(28.0)$ & $75(22.6)$ & $262(26.2)$ & $4(2.6)$ \\
Coagulation Modifiers & $70(5.4)$ & $23(7.4)$ & $17(4.0)$ & $75(5.5)$ & $7(4.5)$ \\
Gl Agents & $134(13.4)$ & $34(14.2)$ & $50(16.9)$ & $210(21.2)$ & $5(3.2)$ \\
Metabolic & $270(24.89)$ & $84(35.8)$ & $109(31.3)$ & $413(38.7)$ & $13(8.3)$ \\
Psychotherapeutic & $125(13.8)$ & $30(16.8)$ & $33(13.7)$ & $152(17.4)$ & $1(0.6)$ \\
Respiratory & $84(8.8)$ & $18(7.9)$ & $51(10.5)$ & $109(11.2)$ & $3(1.9)$ \\
Miscellaneous & $112(12.1)$ & $39(19.5)$ & $56(19)$. & $185(19.1)$
\end{tabular}

Number of self-reported medications ${ }^{2} \quad 1.98(0.69,3.83) \quad 2.50(1.11,3.88)$

$2.08(0.95,3.65) \quad 2.2(1.00,3.96) \quad 0(0.00,0.30)$

${ }^{1}$ Abbreviations: MDS = multiple dietary supplement use; LTMDS = long-term multiple dietary supplement use. ${ }^{2}$ Median $\left(25^{\text {th }}, 75^{\text {th }}\right.$ percentiles $)$

of multiple dietary supplement products. The initial intent of this study was to follow-up the entire sample of participants in the initial LTMDS study of Block and colleagues [7]. However, it was only possible to get follow-up interviews on 235 of the 278 participants (85\%) in the initial LTMDS study. Although the returning LTMDS users who participated in this follow-up study appeared to be in good metabolic health relative to a nationally representative sample of individuals who did not use dietary supplements, we could not determine the health or vital status on the $15 \%$ of the sample that we did not study.

There are additional limitations to the present study and its findings. The LTMDS groups differed in many aspects (such as race, ethnicity, income, education, smoking, dietary supplement use) from the general US population, even after we restricted and stratified in selection of the NHANES sample based on the LTMDS sample characteristics to make it more comparable to the LTMDS participants. We also attempted to account for many of those differences in our statistical analyses, but it is possible that we have not identified or accounted for important differences in health determinants between the LTMDS sample and the general US population. While we also restricted on age, as the age range of the LTMDS users was 50 to 89 years (mean age 72 years), we restricted our NHANES participants to be 40 years or above for comparison to the LTMDS sample because of the NHANES sampling scheme and associated weighting. This resulted in different mean ages in the LTMDS users and the NHANES comparison groups with the mean ages ranging from 54 years in the MVMS users to 60 years in the MDS users. The lower mean ages in the NHANES groups might also be a consequence of the truncation of the age range at 80 years in the NHANES for participants who were older than 80 years [28]. Although we adjusted for age in the statistical models, there may have been some residual effect of this age difference. However, any residual confounding by age would likely result in even greater beneficial differences in cardiometabolic risk for the LTMDS users, as they were older than the NHANES comparison groups.

We must also consider the possibility that supplement use may be a simple marker for a healthy lifestyle. This limitation affects not only the comparison of the LTMDS users to the NHANES NS and MDS groups but also the comparisons between the NHANES supplement use groups and the NS group. We attempted to account for possible confounding by adjustment for important factors such as age, education, income and BMI, which are associated with lifestyle, but the many important lifestyle factor such as physical activity were not available in the LTMDS cohort. Thus, we cannot rule out residual confounding based on lifestyle differences between individuals who use and do not use dietary supplements. Given the diverse nature of the dietary supplements consumed by the LTMDS and NHANES MDS groups and the number of vitamins and minerals in the MVMS group, we cannot identify which products may be associated with the healthier metabolic profiles in these groups relative to the NS group. Finally, the frequency of medication use was lower in the LTMDS group than all of the NHANES supplement groups; however, the different manner in which medication use was prompted for in the respective studies could have resulted in a lower observed prevalence of medication use in the LTMDS participants.

We have examined the metabolic health biomarker profiles for a well-documented cohort of LTMDS users who used MDS for 30 years or more, and demonstrated that some, but not all, of the metabolic benefit associated with the LTMDS in an earlier examination of this cohort persisted with age over an additional 10 year follow-up. The LTMDS and MDS users' overall metabolic health profiles were also better than a representative sample of the US population from NHANES who were not currently using any 
dietary supplements. There were also beneficial associations observed between biomarkers of cardiometabolic health and supplement use among the NHANES SS and MVMS users relative to those not using dietary supplements. These findings add to evidence that use of dietary supplements, particularly MDS use, may provide cardiometabolic health benefits resulting in a lower risk of diabetes and cardiovascular disease.

\section{References}

1. Kantor ED, Rehm CD, Du M, White E, Giovannucci EL. Trends in dietary supplement use among US adults from 1999-2012. JAMA. 2016;316:1464-74.

2. Li C, Hansen RA, Chou C, Calderon Al, Qian J. Trends in botanical dietary supplement use among US adults by cancer status: The National Health and Nutrition Examination Survey, 1999 to 2014. Cancer. 2018;124:1207-15.

3. Bailey RL, Gahche JJ, Miller PE, Thomas PR, Dwyer JT. Why US adults use dietary supplements. JAMA Intern Med. 2013;173:355-61.

4. Ronis MJJ, Pedersen KB, Watt J. Adverse effects of nutraceuticals and dietary supplements. Annu Rev Pharmacol Toxicol. 2018;58:583-601.

5. Mozaffarian D, Forouhi NG. Dietary guidelines and health-is nutrition science up to the task? BMJ. 2018;360:k822.

6. Penders B, Wolters A, Feskens EF, Brouns F, Huber M, Maeckelberghe ELM, et al. Capable and credible? Challenging nutrition science. Eur J Nutr. 2017;56:2009-12.

7. Block G, Jensen CD, Norkus EP, Dalvi TB, Wong LG, McManus JF, et al. Usage patterns, health, and nutritional status of long-term multiple dietary supplement users: a crosssectional study. Nutr J. 2007;6:30. https://doi.org/10.1186/ 1475-2891-6-30

8. National Health and Nutrition Examination Survey. About the National Health and Nutrition Examination Survey [Internet]. 2017 Sep [cited 2019 Sep 17]. Available from: https://www. cdc.gov/nchs/nhanes/about_nhanes.htm

9. Allain CC, Poon LS, Chan CS, Richmond W, Fu PC. Enzymatic determination of total serum cholesterol. Clin Chem. 1974;20:470-5.

10. Roschlau P, Bernt E, Gruber W, [Enzymatic determination of total cholesterol in serum (author's transl)]. Z Klin Chem Klin Biochem. 1974;12:403-7.

11. Trinder P. Determination of glucose in blood using glucose oxidase with an alternative oxygen acceptor. Ann Clin Biochem. 1969;6:24-5.

12. Bucolo G, David H. Quantitative determination of serum triglycerides by the use of enzymes. Clin Chem. 1973;19:476-82.

13. Friedewald WT, Levy RI, Fredrickson DS. Estimation of the concentration of low-density lipoprotein cholesterol in plasma, without use of the preparative ultracentrifuge. Clin Chem. 1972;18:499-502.

14. Babson AL. The DPC Cirrus IMMULITE automated immunoassay system. J Clin Immunoassay. 1991;14:83-8.

15. National Health and Nutrition Examination Survey. 20072008 Data Documentation, Codebook, and Frequencies. Prescription Medications (RXQ_RX_E) [Internet]. 2010 Apr [cited 2019 Sep 17]. Available from: https://wwwn.cdc.gov/Nchs/ Nhanes/2007-2008/RXQ_RX_E.htm

16. National Health and Nutrition Examination Survey. 19882014 Data Documentation. Prescription Medications - Drug
Information (RXQ_DRUG) [Internet]. 2007 Aug [cited 2019 Sep 17]. Available from: https://wwwn.cdc.gov/Nchs/Nhanes/ 1999-2000/RXQ_DRUG.htm\#Component_Description

17. Del Gobbo LC, Imamura F, Aslibekyan S, Marklund M, Virtanen JK, Wennberg M, et al. Omega-3 polyunsaturated fatty acid biomarkers and coronary heart disease: pooling project of 19 cohort ctudies. JAMA Intern Med. 2016;176:1155-66.

18. Rautiainen S, Rist PM, Glynn RJ, Buring JE, Gaziano JM, Sesso HD. Multivitamin use and the risk of cardiovascular disease in men. J Nutr. 2016;146:1235-40.

19. Rautiainen S, Akesson A, Levitan EB, Morgenstern R, Mittleman MA, Wolk A. Multivitamin use and the risk of myocardial infarction: a population-based cohort of Swedish women. Am J Clin Nutr. 2010;92:1251-6.

20. Bailey RL, Fakhouri TH, Park Y, Dwyer JT, Thomas PR, Gahche JJ, Miller PE, et al. Multivitamin-mineral use is associated with reduced risk of cardiovascular disease mortality among women in the United States. J Nutr. 2015;145:572-8.

21. Pittas AG, Chung M, Trikalinos T, Mitri J, Brendel M, Patel K, et al. Systematic review: Vitamin D and cardiometabolic outcomes. Ann Intern Med. 2010;152:307-14.

22. Leermakers ET, Darweesh SK, Baena CP, Moreira EM, Melo van Lent $\mathrm{D}$, Tielemans MJ. The effects of lutein on cardiometabolic health across the life course: a systematic review and meta-analysis. Am J Clin Nutr. 2016;103:481-94.

23. Guallar E, Stranges S, Mulrow C, Appel LJ, MIller ER. Enough is enough: Stop wasting money on vitamin and mineral supplements. Ann Intern Med. 2013;159:850-1.

24. Stanton RA. Supplements and cardiovascular health. Heart Lung Circ. 2015;24:736-9.

25. Hennekens CH, Buring JE, Manson JE, Stampfer M, Rosner B, Cook NR, et al. Lack of effect of long-term supplementation with beta carotene on the incidence of malignant neoplasms and cardiovascular disease. N Engl J Med. 1996;334: $1145-9$.

26. Age-Related Eye Disease Study Research Group. The AgeRelated Eye Disease Study (AREDS): design implications. AREDS report no. 1. Control Clin Trials. 1999;20:573-600.

27. Albert CM, Cook NR, Gaziano JM, Zaharris E, MacFayden J, Danielson E, et al. Effect of folic acid and B vitamins on risk of cardiovascular events and total mortality among women at high risk for cardiovascular disease: a randomized trial. JAMA. 2008;299:2027-36.

28. Johnson CL, Paulose-Ram R, Ogden CL, Carroll MD, KruszonMoran D, Dohrmann SM, et al. National Health and Nutrition Examination Survey: Analytic guidelines, 1999-2010. National Center for Health Statistics. Vital Health Stat. 2013;2(161): $1-24$.

\section{History}

Received September 13, 2020

Accepted February 1, 2021

Published online March 1, 2021

\section{Acknowledgements}

The authors would like to acknowledge the efforts of Drs. Bruce Daggy, Sonhee C. Park, Jamie McManus, and Francis Lau for making this project possible.

\section{Conflicts of interest}

The authors declare no conflicts of interest.

\section{Authorship}

Paul Jacques: Conceptualization, Methodology, Writing - Original Draft Preparation, Writing - Review \& Editing, Supervision, Project 
Administration, Funding Acquisition, Final Approval for Publication. Gail Rogers: Methodology, Formal Analysis, Data Curation, Writing - Original Draft Preparation, Writing - Review \& Editing, Final Approval for Publication.

\section{Funding}

This work was supported by a grant from the Shaklee Corporation and the U.S. Department of Agriculture - Agricultural Research Service (ARS), Agreement No. \#58-1950-4-003. Shaklee Corporation was responsible for the collection of the data used for this project through a contract with MB Clinical Research \& Consulting. Shaklee Corporation was not involved in the analysis and interpretation of data or in the writing of the report. The views expressed in this article are of those of the authors and do not necessarily represent the views of the funding organizations.

\section{ORCID}

Paul F. Jacques

(iD https://orcid.org/0000-0001-5567-3147

\section{Paul F. Jacques}

Tufts University

711 Washington Street

Boston, MA 02111, USA

paul.jacques@tufts.edu 